\title{
El estilo de comunicación del entrenador y la percepción del clima motivacional generado por los entrenadores y compañeros
}

\author{
Coaches' communication style and the perception of the \\ motivational climate created by coaches and teammates
}

\section{O estilo de comunicaçáo do treinador e a percepçáo do clima motivacional gerado por treinadores e colegas}

\author{
Maurício Marques ${ }^{1}$, Roberto Nonohay ${ }^{2}$, Silvia Koller ${ }^{2}$, Gustavo Gauer $^{2}$ y Jaume Cruz ${ }^{1}$ \\ ${ }^{1}$ Universitat Autònoma de Barcelona (UAB) - España y ${ }^{2}$ Universidade Federal do Rio Grande do Sul (UFRGS) - Brasil
}

Resumen: El presente estudio se propuso evaluar las relaciones entre el estilo de comunicación del entrenador y el clima motivacional generado por él y por los compañeros de equipo. Se observó a tres entrenadores de fútbol con el Sistema de Evaluación de las Conductas del Entrenador (CBAS) en tres partidos cada uno. También participaron 39 jugadores de los mismos equipos que los entrenadores, en la franja de edad de 12 a 14 años, que contestaron los cuestionarios: a) cuestionario de percepción de los comportamientos del entrenador (CBAS-PBS); b) clima motivacional generado por el entrenador (PMCSQ-2); c) cuestionario del clima motivacional de los iguales en el deporte (PeerMCYSQ). A partir de los resultados obtenidos, se evidencia una correlación entre conductas positivas que perciben que emiten los entrenadores y la percepción de un clima de implicación a la tarea. Además, de una correlación entre el clima de implicación a la tarea generado por el entrenador y el generado por los compañeros. También se encontró una correlación positiva entre las percepciones de comportamientos punitivos del entrenador y la generación de un clima motivacional de implicación al ego. El clima de implicación al ego generado por el entrenador también se correlaciona con los dos factores del clima de implicación al ego de los compañeros, la competencia y el conflicto intra-equipo.

Palabras clave: Estilo de comunicación, Clima motivacional, Entrenador, Compañeros, Teoría de metas de logro.

Abstract: Our study aims to research the relations between coaches' communication styles and the player's perception regarding the motivational climate generated by the coach and also by their teammates. Three soccer coaches were observed during three games using the Coaches Behaviour Assessment System (CBAS). Also, 39 players from the same teams as the coaches aged from 12 to 14 years participated answering questionnaires regarding a) perception of the coaches' behaviour (CBAS-PBS); b) perception of the motivational climate generated by the coach (PMCSQ-2); c) perception of the motivational climate generated by the teammates (PeerMCYSQ).
We found correlations between positive behaviors from the coaches and the perception of the task involving motivational climate created by them. Moreover, we found a positive correlation between the perception of coaches' punitive behaviors and the generation of an ego involving motivational climate. This also correlates with the idea that teammates create an ego in volving motivational climate when competition and conflicts within group prevail.

Keywords: Communication style, Motivational climate, Coach Trainning, Teammates, Achievement goal theory.

Resumo: Nosso estudo tem por objetivo pesquisar relaçôes entre o estilo de comunicação de treinadores e a percepção de jogadores sobre o clima motivacional gerado pelo treinador e também por seus colegas de equipe. Para tanto, foram observados três treinadores de futebol com o Sistema de Avaliação das Condutas do Treinador (CBAS) em três jogos cada um. Também participaram do estudo 39 jogadores das mesmas equipes que os treinadores, com idades entre 12 e 14 anos, que responderam aos questionários de a) percepção do comportamento do treinador (CBAS-PBS); b) percepçáo do clima motivacional gerado pelo treinador (PMCSQ-2); c) percepção do clima motivacional gerado por colegas de equipe (PeerMCYSQ). Foram encontradas correlaçôes entre as condutas positivas dos treinadores e a percepção de um clima motivacional orientado à tarefa criado por eles. Além de uma correlação entre clima de implicação à tarefa gerado pelo treinador e gerado por colegas. Também encontrou-se uma correlação positiva entre a percepção de que comportamentos punitivos dos treinadores e a geração de um clima motivacional orientado ao ego. O clima de implicação ao ego gerado pelo treinador ainda se correlaciona os dois fatores do clima de implicação ao ego dos colegas, competiçáo e conflito intragrupos.

Palavras-chave: Estilo de comunicação, Clima motivacional, Treinador, Colegas, Teoria do estabelecimento de metas.

\section{Introducción}

La influencia del entrenador deportivo en el logro de experiencias positivas de los jóvenes bajo su tutela a través de

Dirección para correspondencia [Correspondence address]: Maurício Pinto Marques. Facultat de Psicologia. Dpt de Psicologia Bàsica, Evolutiva i de l’Educació. Universitat Autònoma de Barcelona. 08193 Bellaterra, Barcelona (Espańa).E-mail: mauriciopmarques@gmail.com actividades organizadas ha sido un tópico recurrente en la psicología del deporte (Horn, 2008). Se afirma que el deporte tiene implicaciones en la salud física, psicológica, y en el desarrollo moral de sus participantes, que son afectados positiva o negativamente por aquellos con quienes conviven directamente en entrenamientos y partidos, como el entrenador y los 
compañeros de equipo (Holt y Neely, 2011).Nuestro objetivo es investigar si la comunicación percibida por deportistas está acorde con el estilo que observamos en sus entrenadores, y si esta percepción se relaciona de alguna forma con un clima motivacional de implicación a la tarea o al ego generado por el entrenador y por los compańeros de equipo.

Las investigaciones en psicología sobre iniciación deportiva de finales del siglo pasado dejaron de centrarse solamente en cómo obtener un mejor rendimiento de los practicantes y empezaron a ocuparse en cómo asesorar la actuación de los entrenadores (Sousa, Cruz, Torregrosa, Vilches y Viladrich, 2006). Así, a partir de la teoría de Bandura (Singer, Hausenclas y Janelle, 2001) el equipo liderado por el profesor Ronald Smith en la Universidad de Washington creó el Coach Effectiveness Training (CET; Smith, Smoll y Curtis 1979). Con este fin, implantaron un sistema de evaluación de la conducta del entrenador (Smith, Smoll y Hunt, 1977), el Coaching Behavior Assesment System(CBAS) que permite la observación, codificación, registro y análisis de la actuación del entrenador durante situaciones deportivas reales y naturales, como partidos y entrenamientos (Cruz, Torregrosa, Sousa, Mora y Viladrich, 2011). A partir del registro de las conductas reactivas y espontáneas que emiten los entrenadores mientras actúan, se definen sus perfiles de estilo de comunicación, fundamentados en las dimensiones de apoyo, instrucción y punición (Torregrosa, Cruz, Sousa, Viladrich y Villamarín, F 2008).

El CET es un programa cognitivo-conductual de soporte a la formación de entrenadores de iniciación y formación deportiva que procura dar líneas de orientación específicas sobres sus estilos de comunicación (Cruz, Dias, Gomes, Alves, Sá, Viveiros, Almeira y Pinto, 2001). De este modo, la formación del CET se ocupa en asesorar a los entrenadores sobre sus perfiles comunicacionales y les enseña a tener un enfoque más positivo, utilizando el apoyo, el refuerzo, y menos negativo, evitando el castigo y las críticas de los errores. Investigaciones en equipos con entrenadores que se han formado en el CET han comprobado que el enfoque positivo en el deporte infantil genera mayor bienestar, mejor autoestima, promueve mayor aprendizaje de habilidades motoras, además de reducir el miedo al error y la ansiedad deportiva en comparación con otros entrenadores del grupo control que no recibió formación en el CET (Smoll y Smith, 2009).

La evolución del CET hacia el Mastery Approach to Coaching (MAC) considera que el entrenador puede generar un clima motivacional de implicación a la tarea o al ego en los equipos, que afecta el modo en que un joven valora su práctica y a sus compañeros (Duda y Balaguer, 2007; Torregrosa, Cruz, Sousa, Viladrich y Villamarín, 2008). El clima motivacional generado por el entrenador hace referencia a la percepción que tienen los jugadores del modo en que este programa las actividades, gestiona al grupo dándole oportunidades y autoridad, y cómo se comunica, facilitando re- troalimentación en función del rendimiento y esfuerzo en la práctica (Horn, 2011).

Basado en la Teoría de Metas de Logro (Achievement Goal Theory, Nicholls 1984; 1989) un clima motivacional puede ser de implicación a la tarea, donde el equipo tiene como principal objetivo el aprendizaje y el esfuerzo, o hacia el ego, en el que lo más importante es la competencia y la victoria. Además, el clima motivacional general percibido en el ambiente deportivo es influenciado también por otras personas significativas, como padres, directivos y compañeros. En la primera etapa de la adolescencia la influencia de los iguales crece, y comparte protagonismo con el entrenador como principal agente de influencia por la percepción del clima motivacional en el ambiente (Moreno, Conte, Martínez, Alonso, González-Cutre y Cervelló, 2011; Ntoumanis, Vazou y Duda, 2007).

Diversos estudios han revelado la importancia de la actuación del entrenador en la generación de uno u otro clima motivacional, y las ventajas asociadas a un estilo de comunicación más positivo que se correlaciona con un clima motivacional de implicación hacia la tarea (Boixadós, Cruz, Torregrosa y Valiente, 2004; Cecchini, González y Montero, 2007; Smoll y Smith, 2009). Entre las ventajas están una orientación motivacional personal a la tarea en los deportistas, un compromiso mayor, con bajo abandono de un equipo o deporte, mayor satisfacción en la práctica y mejor percepción de habilidad, conjuntamente con más actitudes de fair-play y una ansiedad deportiva menor (Smith y Smoll, 1997; Smith, Smoll y Cumming, 2007). Lo inverso ocurre cuando un entrenador tiene un enfoque de comunicación negativo, que también se correlaciona con la percepción de un clima motivacional de implicación hacia el ego.

Teniendo en cuenta esas investigaciones se han propuesto programas de intervención como el Programa de Asesoramiento Personalizado a Entrenadores (PAPE) (Sousa et al., 2006; Sousa, Smith y Cruz, 2008), que tiene como base la evolución del CET, el MAC. Estos programas buscan la promoción de un estilo de comunicación positivo, así como la promoción de estrategias motivacionales orientadas a la tarea (Cervelló, Moreno, del Villar y Reina, 2007). Un entrenador que ha tenido formación con fundamentos del MAC trabaja, sobre todo, priorizando la motivación, el esfuerzo y el aprendizaje de los jóvenes bajo su tutela, facilitando experiencias deportivas positivas que perdurarán a lo largo de la vida de sus deportistas (Marques, Sousa y Cruz, 2013).

En una revisión sobre el clima motivacional generado por el entrenador, Duda y Balaguer (2007) relacionan la percepción del clima motivacional de implicación a la tarea a una mayor cohesión social en sus equipos, además de fomentar relaciones positivas entre compañeros de equipo. Finalmente, Vazou, Ntoumanis y Duda (2006) encontraron correlaciones positivas entre la percepción del clima motivacional de implicación hacia la tarea generada por el entrenador con un clima 
de implicación hacia la tarea entre iguales. Climas motivacionales que igualmente correlacionaron en negativo con una percepción de un clima motivacional de implicación hacia el ego creado por el entrenador y generado en el equipo.

Además, las investigaciones revisadas estudiaron la relación de otras variables con estilo de comunicación y clima motivacional generado por el entrenador. También investigaron las relaciones entre el clima motivacional generado por los entrenadores y compañeros con otros constructos como el compromiso deportivo, la diversión o el género (Torregrosa et al., 2011). Estos estudios nos llevan a la hipótesis de que entrenadores que emplean un enfoque positivo de comunicación son percibidos como reforzadores y no punitivos por sus deportistas, que perciben que él crea un clima motivacional de implicación a la tarea, lo que también prevalece entre los compañeros de equipo. Así, nuestro objetivo es investigar cómo actúan entrenadores de fútbol infantil del área de Barcelona a partir de la observación de sus estilos de comunicación y de cómo son percibidos por sus equipos. Además, verificar si hay relaciones entre este estilo de comunicación y el clima motivacional que los jóvenes creen que es generado por su entrenador y sus compañeros.

\section{Método}

\section{Participantes}

Participaron en la investigación tres equipos de fútbol infantil de la ciudad de Barcelona, sus tres entrenadores con edades comprendidas entre 24 y 45 años $(M=32.3 \mathrm{DE}=11.15)$ además de los jugadores de esos equipos, 39 jóvenes deportistas entre 12 y 14 ańos $(\mathrm{M}=13.1 \mathrm{DE}=0.36)$. Todos competían en el campeonato de la liga Catalana de la categoría infantil, de primera y segunda división, considerada con un nivel competitivo alto dentro su franja de edad.

\section{Instrumentos}

Estilo de comunicación del entrenador. Se utilizó el Cuestionario de Percepción de los Comportamientos del Entrenador (CBAS-PBS) elaborado en inglés por Smith, Smoll y Curtis (1979) conjuntamente con el Sistema de Evaluación de Comportamientos del Entrenador (CBAS) instrumento de observación y codificación de los comportamientos del entrenador en situación de entrenamiento y competición y utilizado en España en numerosos estudios (Cruz, et al.; 2011, Mora, Cruz y Sousa, 2013; Sousa, Smith y Cruz 2008). El CBAS y el CBAS-CPBS se agrupan en tres dimensiones conductuales que determinan el estilo de comunicación del entrenador: a) Apoyo (incluye las Categorías Refuerzo y Ánimo Contingente al Error); b) Instrucciones (incluye Instrucción Técnica al Error e Instrucción Técnica General) y c) Punición (incluye
Castigo e Instrucción Técnica Punitiva). El CBAS evalúa los comportamientos observados del entrenador. El CBASCPBS mide la percepción que tienen los deportistas de la frecuencia con que su entrenador emite dichos comportamientos. Cuenta con 11 ítems representando las tres dimensiones en preguntas como (Ej.: "Os anima vuestro entrenador durante el partido?") o (Ej.: "Se enfada tu entrenador cuando fallas en algo?") que son contestadas en una escala Likert de 7 puntos que va de 0 (nunca) a 6 (siempre). Se obtuvo un $\alpha=0.65$ para la dimensión Apoyo, $\alpha=0.56$ para la dimensión Instrucción y $\alpha=0.41$ para la dimensión Punición, resultados de consistencia interna que no se deben a la ausencia de correlación entre ítems, sino a su reducido número en algunas subescalas.

Clima motivacional generado por el entrenador. Se utilizó la segunda versión del Cuestionario de Percepción del Clima motivacional generado por el entrenador (PMCSQ-2) elaborado originalmente en inglés por Newton, Duda y Yin (2000) y validado en español por Balaguer, Givernau, Duda y Crespo (1997). Este instrumento cuenta con 29 ítems contestados a partir de la frase: "En mi equipo el entrenador..." y divididos en dos subescalas, una de implicación a la Tarea (Ej.“....anima a los jugadores a trabajar sus puntos débiles”) y otra de implicación al Ego (Ej.: “...deja muy claro quiénes son los mejores jugadores”). La subescala de implicación a la Tarea tiene 15 ítems y de implicación al Ego 14 ítems que se contestan a partir de una escala tipo Likert de 6 puntos que va de 1 (muy en desacuerdo) a 5 (muy de acuerdo). Una puntuación más alta en cada subescala se refiere a la percepción que el entrenador genera en el equipo un clima motivacional de implicación a la Tarea o al Ego. En el presente estudio obtuvimos un $\alpha=0.87$ para la subescala de implicación a la Tarea y de $\alpha=0.91$ para la escala de implicación al Ego.

Clima Motivacional promovido por los compańeros. Se utilizó la versión española del Peer-Created Motivational Climate Sport Questionnaire (PeerMCYSQ) un instrumento creado y validado en inglés por Ntoumanis y Vazou (2005) para evaluar el clima motivacional percibido entre los compañeros de equipo. Cuenta con 21 ítems que se agrupan en cinco factores: mejora, apoyo a la relación y esfuerzo (referentes a un clima motivacional de implicación a la tarea) y competición intra-equipo y conflicto intra-equipo (referentes a un clima motivacional de implicación al ego) en su versión original. Sin embargo, Moreno et al. (2011) hicieron un estudio en España donde se constató que un modelo con 3 factores (clima motivacional, tarea y competición y conflicto intra-equipo) se ajusta mejor para la evaluación. A partir de la frase "En este equipo, la mayoría de jugadores..." se contestan frases como (Ej.: "...se ayudan unos a otros para mejorar") o (Ej.: “...critican a sus compańeros cuando cometen errores") en una escala tipo Likert de 7 puntos que va del 1(para nada de acuerdo) al 7 (totalmente de acuerdo). En este 
estudio obtuvimos un $\alpha=0.85$ para el factor tarea, $\alpha=0.53$ en el factor competición y $\alpha=0.64 y$ en el factor conflicto.

\section{Procedimiento}

Al final de temporada, se solicitó a los tres equipos la colaboración en este estudio. Los coordinadores y los entrenadores de cada club dieron su consentimiento para participar en la investigación. Se observaron con el CBAS tres partidos por equipo, teniendo como mínimo observaciones en uno como local y uno como visitante. La administración de los cuestionarios ocurrió después de un entrenamiento en que se pidió a los deportistas que contestasen un cuadernillo con los siguientes cuestionarios: CBAS-PBS, PMCSQ-2 y PeerMCYSQ.

El encuestador estuvo presente todo el tiempo para contestar cualquier duda que pudiera surgir. Las aplicaciones se desarrollaron sin incidentes. Al terminar se agradeció la colaboración de los jugadores, entrenadores y coordinadores y se les indicó que recibirían un informe de los resultados al final del estudio.

\section{Análisis de Datos}

El análisis de datos se realizó mediante el programa estadístico SPSS. La tasa de participación fue de $97.5 \%$ con sólo un cuestionario invalidado sobre un total de 40 recogidos. Se utilizaron técnicas de análisis descriptivo, análisis de fiabilidad mediante el Alpha de Cronbach y los coeficientes de correlación de Pearson. Los datos atienden a las indicaciones de normalidad, analizando kurtosis, asimetría y el gráfico Q-Q.

\section{Resultados}

En esta sección se presentan: a) el porcentaje de las conductas de cada entrenador en los tres partidos observados, agrupadas en las dimensiones de apoyo,instrucción y punición; b) los resultados descriptivos para cada una de las subescalas de los cuestionarios: CBAS-PBS, PMCSQ-2 y PeerMCYSQ; c) las correlaciones entre las subescalas de clima motivacional inducido por el entrenador, clima motivacional percibido entre los iguales y de la percepción del estilo de comunicación del entrenador. a) Resultados de las observaciones de los entrenadores

Figura 1. Resultados de la observación de los entrenadores

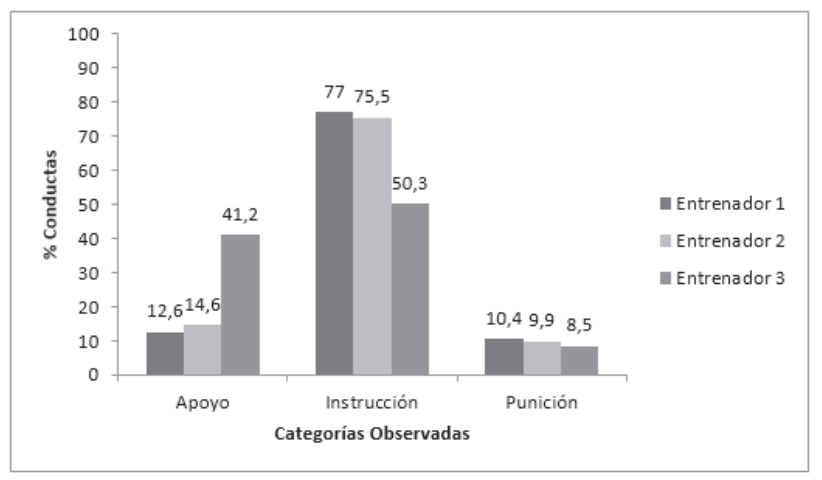

Tal como aparece en la Figura 1, a partir de las observaciones de tres partidos de cada entrenador se obtuvo un perfil conductual por medio del porcentaje que cada dimensión ocupa dentro de sus comunicaciones por partidos. Los entrenadores 1 y 2 presentaron un estilo de comunicación de mucha instrucción $(77 \%$ y 75,5\% del total de comportamientos en cada partido), conductas de apoyo en menor cantidad (12,6\% y $14,6 \%)$ y algunas conductas de punición (10,4\% y 9,9\%). El entrenador 3 demostró ser más reforzador (41,2\% de sus comunicaciones fueron de apoyo), tener la mitad (50,3\%) de sus conductas relacionadas a instrucciones y también emitió menos comportamientos punitivos en sus partidos $(8,5 \%$ del total).

\section{b) Resultados descriptivos para cada una de las subescalas}

Verificamos en la Tabla 1, que los futbolistas perciben que reciben de sus entrenadores un nivel alto de apoyo $(M=4,78)$ y de Instrucciones $(\mathrm{M}=4,60)$ y una menor cantidad de conductas de Punición $(\mathrm{M}=2,23)$. Los jugadores perciben que los entrenadores generan un Clima de Implicación a la Tarea $(\mathrm{M}=4,12)$ superior al Clima de Implicación al Ego $(\mathrm{M}=2,36)$. Los valores medios de la subescalas del Clima Motivacional percibido como generado por los pares se igualan en Tarea $(M=3,97)$ y Conflicto $(M=3,93)$ y es un poco superior en Competición $(\mathrm{M}=4,53)$. 
Tabla 1. Media $(M)$, desviación típica (SD) y rango de las subescalas de Estilo de Comunicación, Climas Motivacionales Entrenador e Iguales

\begin{tabular}{lccc}
\hline \multicolumn{1}{c}{ Escalas } & M & SD & Rango \\
\hline Estilo de Comunicación Percibido & & & \\
Apoyo & 4.78 & 1.15 & $0-6$ \\
Instrucciones & 4.6 & 0.91 & $0-6$ \\
Punición & 2.23 & 1.35 & $0-6$ \\
Clima Motivacional Percibido Entrenador & & & \\
Tarea & 4.12 & 0.55 & $1-5$ \\
Ego & 2.36 & 0.81 & $1-5$ \\
Clima Motivacional Percibido entre Iguales & & & \\
Tarea & 3.97 & 0.97 & $1-7$ \\
Competición & 4.53 & 0.96 & $1-7$ \\
Conflicto & 3.93 & 1.23 & $1-7$ \\
\hline
\end{tabular}

c) Correlaciones entre la percepción de los comportamientos de los entrenadores, clima motivacional generado por entrenadores e iguales

Como se observa en la Tabla 2, la percepción que los jugadores tienen de los comportamientos de apoyo que emite el entrenador se relaciona positivamente con la percepción de que él emite comportamientos de instrucción $(\mathrm{r}=0.4)$ y genera un clima motivacional de implicación a la tarea $(\mathrm{r}=$ 0.62). Además, que un entrenador instructivo correlaciona positivamente con la percepción de un clima motivacional de implicación a la tarea $(r=0.48)$. Por su parte, la percepción de que un entrenador emite conductas de punición correlaciona positivamente con un clima de implicación al ego $(r=0.44)$. Se encontró también una correlación positiva entre la percepción de que los entrenadores generan un clima motivacional al ego con climas motivacionales de competición y conflictos promovidos entre los compańeros de equipo ( $r=0.55 \mathrm{y}$ $\mathrm{r}=0.46$ ), que también se correlacionan positivamente entre sí $(\mathrm{r}=0.57)$.

Tabla 2. Correlaciones entre las subescalas de Estilo de comunicación, Clima motivacional generado por el entrenador y Clima motivacional entre Iguales

\begin{tabular}{|c|c|c|c|c|c|c|c|c|}
\hline Factores & 1 & 2 & 3 & 4 & 5 & 6 & 7 & 8 \\
\hline 1. Apoyo & - & $0.40^{* *}$ & -0.25 & $0.62^{* *}$ & -0.18 & 0.18 & -0.16 & 0.11 \\
\hline 2. Instrucción & & - & $0.40^{*}$ & $0.48^{* *}$ & -0.03 & 0.28 & -0.28 & 0.17 \\
\hline 3. Punición & & & - & -.17 & $0.44^{* *}$ & 0.10 & 0.07 & 0.09 \\
\hline 4. Clima Tarea Entrenador & & & & - & -0.23 & $0.38^{*}$ & -0.31 & -0.12 \\
\hline 5. Clima Ego Entrenador & & & & & - & 0.09 & $0.55^{* *}$ & $0.46^{* *}$ \\
\hline 6. Clima Iguales Tarea & & & & & & - & -0.23 & -0.26 \\
\hline 7. Clima Iguales Competición & & & & & & & - & $0.57^{* *}$ \\
\hline 8. Clima Iguales Conflicto & & & & & & & & - \\
\hline
\end{tabular}

**. La correlación es significativa en un nivel 0,01 (bilateral).

*. La correlación es significativa en un nivel 0,05 (bilateral).

\section{Discusión}

Este estudio tiene como objetivo conocer las posibles relaciones entre el estilo de comunicación de entrenadores y la percepción del clima motivacional generado por entrenadores y compañeros. A partir de la observación de las conductas de los entrenadores, se encontró un predominio de conductas de instrucción, seguida por una frecuencia menor de conductas de Apoyo, y menor aún de conductas de punición. Esos perfiles van de acuerdo con los observados por Sousa et al. (2006) en una intervención con el PAPE. El entrenador 3, que se destacó por ser el más reforzador del estudio, emitió una menor frecuencia de instrucción que ellos. El hecho de que dicho entrenador estudiara educación infantil puede ser el motivo por el cual supera el promedio de los entrenadores en apoyo.
La percepción del estilo de comunicación positivo que los jugadores tienen de sus entrenadores es todavía superior a la observada, pues la media de percepción de conductas de apoyo es equivalente a media la de instrucciones (Tabla 1). Eso puede ser explicado porque ambas se relacionan entre sí, y también con la percepción de un clima motivacional generado por el entrenador de implicación a la tarea. Así, un entrenador que apoya e instruye es percibido como alguien interesado en el aprendizaje y la diversión de los futbolistas, resultado similar al encontrado por Torregrosa et al. (2008). Lo mismo sucede con los bajos promedios de conductas de punición observados y percibidos, que a su vez correlacionan positivamente con la percepción de un clima motivacional de implicación al ego. Clima motivacional que tuvo el promedio por debajo del clima motivacional hacia la tarea,. Este perfil 
con enfoque positivo es conocido también como "entrenador efectivo" (Horn, 2008).

La percepción de la generación de climas de implicación a la tarea y al ego no correlacionaron entre sí negativamente, tanto para entrenadores como para iguales, como en los resultados de Boixadós et al. (2004) donde se sugiere que los dos climas pueden coexistir simultáneamente en un contexto dado. Las subescalas de implicación al ego del cuestionario del clima motivacional entre iguales correlacionan positivamente con la percepción del clima motivacional hacia el ego generado por el entrenador, sugiriendo que la competición y el conflicto entre compañeros, también es motivada por el entrenador como se sugiere en Ntoumanis et al. (2007). Además, coexiste con la percepción del clima hacia la tarea generado por el responsable, que a la vez que enseña y apoya, también refuerza la competitividad entre los futbolistas, algo intrínseco al deporte en una liga competitiva como la de nuestros participantes. Posiblemente por eso los promedios entre los tres factores del clima motivacional percibido entre iguales están cercanos, aunque sólo los factores de competición y conflicto, que forman el clima motivacional de implicación al ego, correlacionen ente sí.

Nuestros resultados confirman que los entrenadores, aunque tengan un enfoque positivo, deben prestar atención al clima motivacional que puede existir entre compañeros, pues los resultados de este estudio sugieren que incluso en equipos con entrenadores reforzadores y poco punitivos puede darse la percepción de un clima motivacional de implicación al ego. En el mismo sentido, en equipos de deportistas jóvenes, sobre todo adolescentes, un clima de competición y conflicto entre compañeros puede existir independientemente del entrenador, lo que trae nuevos retos para los responsables en gestionar a estos jóvenes. Aunque esos factores sean parte del fútbol competitivo y de la adolescencia en sí, hay que resaltar que lo más importante es el aprendizaje y la diversión de todos, aspecto en el que puede influir el entrenadora través de sus charlas y, principalmente, de las conductas y actitudes en los partidos y entrenamientos (Horn, 2008).

Nuestro estudio aporta más datos en algunos temas que ya describieron Horn (2008) y Ntoumanis et al. (2007) en sus trabajos sobre entrenadores efectivos y clima motivacional de los pares o compañeros de equipo, respectivamente, aunque presenta limitaciones. La principal fue el tamańo de muestra, que no ha permitido análisis estadísticos como la regresión, que posibilitaría analizar qué parte del clima motivacional de implicación al ego dentro del equipo puede ser explicado por la percepción del clima generado por el entrenador o la percepción de su estilo de comunicación. Otra limitación importante es el sesgo que pueden haber sufrido los datos observacionales, pues fueron recogidos por un solo investigador, aunque fue entrenado en el método de observación con el CBAS. El hecho de que todos los participantes sean del sexo masculino también debe ser considerado como una limitación para la generalización de los resultados.

\section{Aplicaciones prácticas}

Los resultados de nuestro estudio refuerzan la importancia de las conductas de apoyo y la generación de un clima motivacional de implicación a la tarea por parte de los entrenadores, como se ha estudiado ampliamente en la última década. Sin embargo, se sugiere la necesidad de que padres, directivos y entrenadores, permanezcan atentos al clima motivacional que existe entre los jugadores de sus equipos. Aunque un entrenador tenga un perfil positivo, el clima del equipo puede influir negativamente en la experiencia deportiva de los jóvenes, generando ansiedad, baja autoestima y malestar, llevando incluso al abandono deportivo. Sugerimos estudios con más participantes que permitan correlacionar el estilo de comunicación del entrenador con la percepción del clima motivacional generado por el entrenador y compañeros. Asimismo, nuestra investigación ha podido aportar nuevas ideas en relación a las variables trabajadas, que suelen ser investigadas por separado. Fomentamos más investigaciones de carácter práctico, para que el deporte sea fuente de experiencias sanas y positivas para los jóvenes, principalmente a partir de las conductas de los adultos responsables de ellos, los entrenadores.

\section{Referencias}

1. Balaguer, I. Givernau, M., Duda, J.L., y Crespo, M. (1997). Análisis de la validez de constructo y de la validez predictiva del cuestionario de clima motivacional percibido en el deporte (PMCSQ-2) con tenistas españoles de competición. Revista de Psicología del Deporte, 11, 41-58.

2. Boixadós, M., Cruz, J., Torregrosa, M., y Valiente, L. (2004). Relationships among motivational climate, satisfaction, perceived ability, and fair play attitudes in young soccer players. Journal of Applied Sport Psychology, 16, 301-317.

3. Cecchini, J.A., González, C., y Montero, J. (2007). Participación en el deporte y fair play. Psicothema, 19, 57-64.

4. Cervelló, E., Moreno, J.A., del Villar, F., y Reina, R. (2007). Desarrollo y validación de un instrumento de medida de las estrategias motiva- cionales empleadas en las clases de educación física. Revista Iberoamericana de Psicología del Ejercicio y el Deporte, 2(2), 53-72.

5. Cruz, J.F., Dias, C., Gomes, R., Alvez, A., Sá, S., Viveiros, I., Almeida, S., y Pinto, S. (2001). Um programa de formação para a eficácia dos treinadores da iniciação e formação desportiva. Análise Psicológica, 19, 171-182.

6. Cruz, J., Torregrosa, M., Sousa, C., Mora, A., i Viladrich, C. (2011). Efectos conductuales de programas personalizados de asesoramiento a entrenadores en estilo de comunicación y clima motivacional. Revista de Psicología del Deporte.20(1), 179-195.

7. Duda, J. L., y Balaguer, I. (2007). Coach-created motivational climate. In: Jowett, S. y Lavalle, D. (Eds.), Social Psychology in Sport (pp. 117130). Champaing, IL: Human Kinetics. 
8. Holt, N. L., y Neely, K. C. (2011). Positive youth development through sport: a review. Revista de iberoamericana de psicología del ejercicio y el deporte, 6 (2), 299-316.

9. Horn, T.S. (2008). Coaching effectiveness in the sport domain. In T.S. Horn (Ed.), Advances in sport psychology (pp. 239-268). Champaign, IL: Human Kinetics

10. Marques, M. P., Sousa, C., y Cruz, J. (2013) Estrategias para la enseñanza de competencia de vida a través del deporte en jóvenes en riesgo de exclusión social. Apunts Educación Física y Deportes, 112, 63-71.

11. Mora, A., Cruz, J., y Sousa, C. (2013). Como mejorar el clima motivacional y los estilos de comunicación en el ámbito de la Educación Física y el deporte. Infancia y Aprendizaje, 36 (1), 91-103

12. Moreno, J. A., Conte, L., Martínez, C. Alonso, N., González-Cutre, D., y Cervelló, E. (2011). Propiedades psicométricas del Peer Motivational Climate in Youth Sport Questionnaire (PeerMCYSQ) con una muestra de deportistas españoles. Revista de Psicología del Deporte, 20(1), 101-118

13. Newton, M., Duda, J.L., y Yin, Z.N. (2000). Examinaton of the psychometric properties of female athletes. Journal of Sport Sciences, 18, 275-290.

14. Nicholls, J. (1984). Achievement Motivation: Conceptions of Ability, Subjective Experience, Task Choice, and Performance. Psychological Review, 91 (3), 328-346.

15. Nicholls, J. (1989). The competitive ethos and democratic education. Cambridge, MA: Harvard University Press.

16. Ntoumanis, N. y Vazou, S. (2005). Peer motivational climate in youth sport: Measurement development and validation. Journal of Sport and Exercise Psychology, 27, 432-455.

17. Ntoumanis, N., Vazou, S., y Duda, J. L. (2007). Peer-created motivational climate. En S. Jowette y D. Lavallee (Eds.). Social psychology in sport (pp. 145-156). Champaign, IL: Human Kinetics.

18. Singer, R.N., Hausenblas, A. H., y Janelle, C.M. (2001). Handbook of Sport Psychology. New Yorke: John Wiley y Sons.
19. Smith, R.E., y Smoll, F.L. (1997). Coach-mediated team building in youth sports. Journal of Applied Sport Psychology, 9, 114-132.

20. Smith, R.E. y Smoll, F. L. (2007). Social-cognitive approach to coaching behaviors. En S. Jowett, y D. Lavallee (Eds). Social Psychology in Sport (pp.75-90).Champaign IL: Human Kinetics.

21. Smith, R.E. y Smoll, F.L., y Cumming, S.P. (2007). Effects of a motivational climate intervention for coaches on young athletes' sport performance anxiety. Journal of Sport and Exercise Psychology, 29, 39-59.

22. Smith, R.E., Smoll, F.L., y Curtis, B. (1979). Coach effectiveness training. A cognitive behavioral approach to enhancing relationship skills in youth sports coaches. Journal of Sport Psychology, 1, 59-75.

23. Smith, R.E., y Smoll, F.L., y Hunt, E.B. (1977). A system for the behavioral assessment of athletic coaches. Research Quarterly, 48, 401-407.

24. Smoll, F. L. y Smith, R. E. (2009). Claves para ser un entrenador excelente. Barcelona: Inde.

25. Sousa, C., Cruz, J., y Smith, R. (2008). An Individualized Behavioral Goal-Setting Program for Coaches. Journal of Clinical Sport Psychology, 2, 258-277.

26. Sousa, C., Cruz, J., Torregrosa, M., Vilches, D., y Viladrich, C. (2006). Evaluación conductual y programa de asesoramiento personalizado a entrenadores (PAPE) de deportistas. Revista de Psicología del Deporte, $15,263-278$.

27. Torregrosa, M., Cruz, J., Sousa, C., Viladrich, C., y Villamarín, F. (2008). El clima motivacional y el estilo de comunicación del entrenador como predictores del compromiso en futbolistas jóvenes. Psicothe$m a, 20,2,254-259$.

28. Torregrosa, M., Viladrich, C., Ramis, Y., Azócar, F., Latinjak, A. y Cruz, J. (2011). Efectos en la percepción del clima motivacional generado por los entrenadores y compañeros sobre la diversión y el compromiso. Diferencias en función de género. Revista de Psicología del Deporte, 20(1), 243-255.

29. Vazou, S., Ntoumanis, N., y Duda, J.L. (2006). Predicting young athletes' motivational indices as a function of their perceptions of the coachand peer-created climate. Psychology of Sport \& Exercise, 7, 215-233. 